\title{
IMPROVED DISTANCE METRIC TECHNIQUE FOR DERIVING SOFT RELIABILITY INFORMATION OVER RAYLEIGH FADING CHANNEL
}

\author{
0. 0. Ogundile ${ }^{1,}{ }^{*}$, M. O. Oloyede ${ }^{2}$, O. A. Osanaiye ${ }^{3}$ and F. A. Aina ${ }^{4}$
}

1, Dept. of Physics \& TeleCommunications, TAi SOLARIN Univ. of EDUCATION, IJEBU-ODE, OGUn STATE, NiGERIA. 2, DEPT. OF INFORMATION AND COMMUNICATION SCIENCE, UNIVERSITY OF ILORIN, ILORIN, KWARA STATE, NIGERIA.

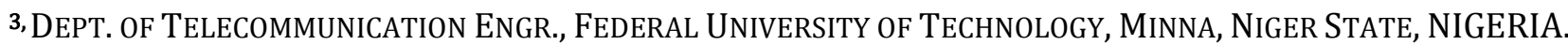
4, DEPARTMENT OF TELECOMMUNICATION SCIENCE, UNIVERSITY OF ILORIN, ILORIN, KWARA STATE, NIGERIA. E-mail addresses:1'ogundileoo@gmail.com, ${ }^{2}$ tahir.oloyede@gmail.com, ${ }^{3}$ opeyemi177@futminna.edu.ng, 4folayo.aina@pgr.anglia.ac.uk

\begin{abstract}
This paper presents an improved Distance Metric (DM) technique for deriving soft reliability information over Rayleigh fading channel. We compared this proposed DM technique with the conventionally used DM technique in the literature. The conventional DM method derives the soft reliability information from the output received symbols while the proposed DM method derives the soft reliability information from the Channel State Information (CSI) which result due to variations in the channel gain. Performance analysis of these DM methods are verified over flat Rayleigh fading channels, and on time-varying frequency-selective Rayleigh fading channels using rectangular M-QAM and OFDM systems respectively. Also, two channel estimation techniques are used to derived the CSI assuming different normalized Doppler frequency and frame length size. The performance of the conventional and proposed soft reliability derivation methods are documented through computer simulations assuming Koetter and Vardy, ReedSolomon (KV-RS) soft decision decoding algorithm as the Forward Error Correction (FEC) scheme. From the computer simulation results, the proposed DM method offers significant improvement in Codeword Error Rate (CER) performance in comparison with the conventional DM method with no significant increase in computational delay and time complexity.
\end{abstract}

Key words: CSI, cubic estimators, DM, fading channels, KV-RS decoder, LMMSE, OFDM, R-matrix, 16QAM.

\section{INTRODUCTION}

Forward Error Correction (FEC) schemes such as ReedSolomon (RS), Turbo and Low Density Parity Check (LDPC) codes have been extensively investigated to provide reliable data transmission [1-6]. Hard decision decoding algorithms were first introduced to improve the performance of receivers in fading channels. Much recent techniques employ soft decision FEC schemes to provide efficient data transmission. With respect to the FEC scheme used, soft-decision decoding requires reliability information from the channel output to correctly decode the data encoded at the transmitter. The soft reliability information can be derived using Log Likelihood Ratio (LLR) or Distance Metric (DM) methods [6-9].

Several works have discussed the conventional DM methods of deriving soft reliability information from the channel output [6-8]. In [6-8], the conventional DM method derives the soft reliability information by measuring the distance from the received signal to the output symbol constellation. Note that the conventional DM method does not consider the fading channel effect (variation in the channel) in deriving the reliability information. As such, this method does not offer an efficient set of soft reliable information to the decoder. In this paper, we propose an improved and efficient DM method of deriving soft reliability information over Rayleigh fading channels with White Additive Gaussian Noise (AWGN). This proposed method considers the effect of the fading channel in deriving the soft reliability information. Thus, the proposed DM method derives the soft reliability information by comparing the distance from the estimated Channel State Information (CSI) to the scaled CSI as shown in Section 4. 
The Koetter and Vardy, Reed-Solomon (KV-RS) soft decision decoding algorithm is chosen as the FEC scheme to verified the performance of this proposed DM method with a fair comparison to the conventionally used DM method. Rectangular $M$-ary Quadrature Amplitude Modulation ( $M$-QAM) is adopted as the modulation scheme over flat Rayleigh fading channels. Also, we adapted $M$-QAM in an OFDM system structure in order to verify the performance of the proposed method on time-varying frequency-selective Rayleigh fading channels.

$M$-QAM and OFDM systems are used in this work because of the numerous advantages they provide in wireless communication applications. Most advantageously, their use in wireless technologies is motivated by the limited transmission bandwidth and high data transmission rate demand. Furthermore, OFDM offers other advantages which include its robustness against multipath fading which is a main contributor of Inter Symbol Interference (ISI). However, the reliable use of M-QAM and OFDM in wireless communication systems require accurate channel estimation techniques because of the varying properties of the channel. This has resulted in extensive research on different optimal channel estimation techniques. In this paper, the CSI is derived using the Pilot Symbol Assisted Modulation (PSAM) Linear Minimum Mean Square Error (LMMSE) criterion $[10,11]$, and the PSAM cubic estimator [12] for different frame/block length $L_{f}$ and normalized Doppler frequency $f_{d} T$. Basically, we derive the CSI using two channel estimation methods in order to give weighty remarks on this proposed method of extracting soft reliability information.

The performance comparison between the proposed and the conventional DM methods is documented through computer simulations. In Section 5.0, Fig. 6 and Fig. 7 verify the performance of both DM methods of extracting soft reliability information on flat Rayleigh fading channels. Likewise, Fig. 8 and Fig. 9 in Section 5. 0 verify their performances on time-varying frequencyselective Rayleigh fading channels. This paper also present a simple analytical computational delay and time complexity between the conventional and the proposed DM methods as shown in Section 5.0.

The relevance and contributions of this paper are as follows. The paper propose an improved method of deriving reliability information in the form of a DM for soft decision FEC schemes over fading channels. More so, we analyse the performance of this proposed DM method for $M$-QAM and OFDM systems combined with
RS codes. $M$-QAM and OFDM systems are used with $R S$ codes in different wireless communication applications which include LTE, DVB, WIMAX, ADSL, DAB, etc. Hence, the findings in this paper is useful to enhance the performance of $M$-QAM and OFDM systems with soft decision FEC schemes on flat Rayleigh fading channels, and on time-varying frequency-selective Rayleigh fading channels respectively.

The rest of this paper is structured as follows: Section 2.0 explains the $M$-QAM and OFDM system models. In section 3.0, we briefly derive the CSI using two different channel estimation methods. The conventional and proposed DM methods of deriving soft reliability information are discussed in Section 4.0. Section 5.0 presents the comparative computer simulation results and discussions. The computational delay and time complexities analysis of the conventional DM method in comparison with the proposed DM method is also discussed in this Section. We summarize the paper with important remarks in Section 6.0.

\section{SYSTEM MODEL}

\section{$2.1 M$-QAM System}

The PSAM $M$-QAM system model is as shown in Fig. 1. The input data is encoded using an (n, k) $R S$ code, where $\mathrm{k}$ and $\mathrm{n}$ are the $R S$ information and code word lengths respectively. This encoded data is mapped to rectangular $M$-QAM complex symbols where the inphase and quadrature parts take values from a set $\left(1_{c}, \pm 3_{c}, \ldots \pm c(m-1)\right)$ [13]. Specifically, 16-QAM $(M$ $=16$ ) is assumed in this work; thus, $m=\sqrt{M}$. Although $M=16$ is used in this paper, the simulation results can be extended to other signal constellations (that is $M=$ $4,8,32,64$, etc.). Pilot symbols $\left(t_{p}\right)$ are uniformly positioned in the mapped data $\left(t_{i}\right)$ as illustrated in Fig. 2 . The paper assumes that all the pilot symbols take on the same complex value. The combined symbol structure $\left(t_{t}\right)$ is divided into frames of the same length $(L)$ with each frame starting with a known pilot symbol $\left(t_{p}\right)$, and subsequently $\left(L_{f}-1\right)$ data symbols. The frame structures are transmitted over a flat Rayleigh fading channel and distorted with Additive White Gaussian Noise (AWGN). The fading behaviour of the channel is a function of the normalized Doppler frequency $\left(f_{d} T\right)$, where $\left(f_{d}\right)$ is Doppler frequency, and $T$ is the symbol period. The Jake's isotropic scattering model as previously presented in [14] is assumed in this paper.

The faded noisy received signal is defined as:

$$
r_{t}=t_{t} \eta_{\mathrm{t}}+\mathrm{Z}_{\mathrm{t}}
$$


where $t_{t}$ is the transmitted pilot and data symbols, $Z_{t}$ is the complex AWGN with $\sigma^{2}=N_{0} / 2$, and $\eta_{\mathrm{t}}$ is the complex zero mean Gaussian variables representing the fading distortion at the pilot and data symbol's position. The CSI is only known at the pilot symbol positions. Therefore, the CSI at the data symbol positions is obtained using the properties of the known pilot symbols.

\subsection{OFDM System}

$M$-QAM is adapted in an OFDM system structure as shown Fig.3 and Fig.4, where Fig.3 represents the OFDM transmitter model and Fig. 4 depicts the OFDM receiver structures. At the transmitter, the combined data sequence $\left(t_{t}\right)$ is also divided into frames of equal length $L_{f}$ as shown in Fig. 5. Note that we assume a block/frame type pilot arrangement in the OFDM structure design. The $t_{t}$ is fed into a serial-to-parallel converter $(S / P)$. The Inverse Discrete Fourier Transform (IDFT) block converts the frequency domain OFDM signal $x(l)$ to the time domain as defined in Eqn. (2) $[10]:$

$$
x(L)=\frac{1}{\sqrt{\lambda}} \sum_{l=0}^{\lambda-1} x(l) e^{+j(2 \pi l L / \lambda)},
$$

where the length of the DFT is denoted as $\lambda$. In order for the length of the carriers to be compatible with the filter size, the carriers are padded in between with a number of zeros. The time domain OFDM signal $x(L)$ is fed into a parallel-to-serial $(P / S)$ converter. To eliminate ISI, a cyclic prefix of $C_{p}$ sample durations is added to the OFDM symbol as defined in Eqn. (3) [10]:

$X(L)= \begin{cases}x(\lambda+L), & L=-C_{p},-C_{p}+1, \cdots,-1, \\ x(L) & L=0,1, \cdots, \lambda-1 .\end{cases}$

After the Digital-to-Analogue Conversion $\left(D_{A C}\right)$ block, the OFDM signal is transmitted over a time-varying frequency-selective Rayleigh fading channel with Additive White Gaussian Noise (AWGN).

Hence, the time domain OFDM signal at the receiver end is given as:

$$
\bar{R}(L)=\bar{X}(L) \otimes \bar{H}(L)+\mathrm{z}(L),
$$

where $\mathrm{Z}(L)$ is the complex AWGN with $\sigma^{2}=N_{0} / 2, \bar{H}(L)$ is the channel impulse response, and $\bar{X}(L)$ is the transmitted OFDM signal.

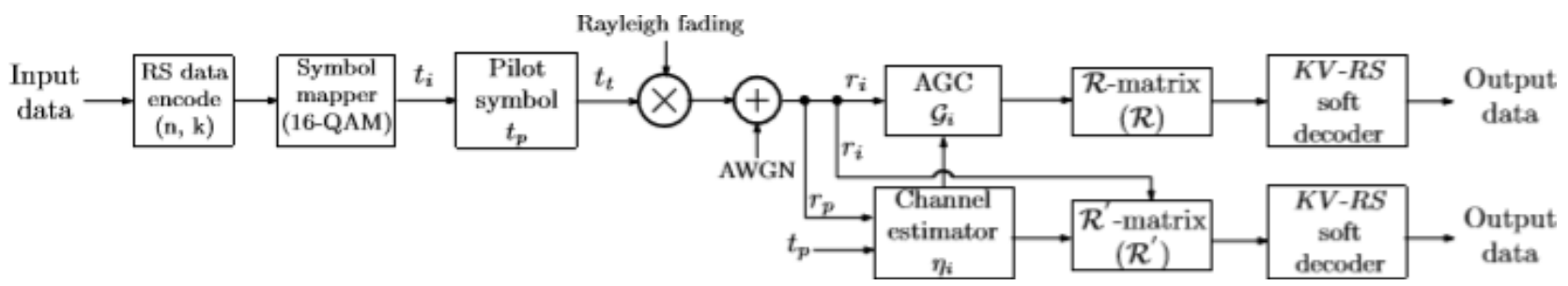

Figure 1: M-QAM system model on flat Rayleigh fading channels

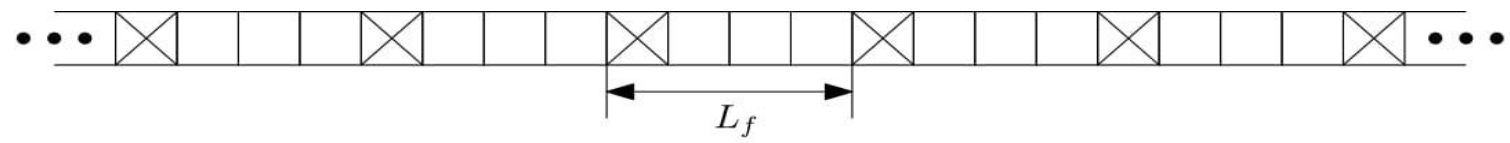

Pilot symbol Data symbol

Figure 2: Pilot arrangement.

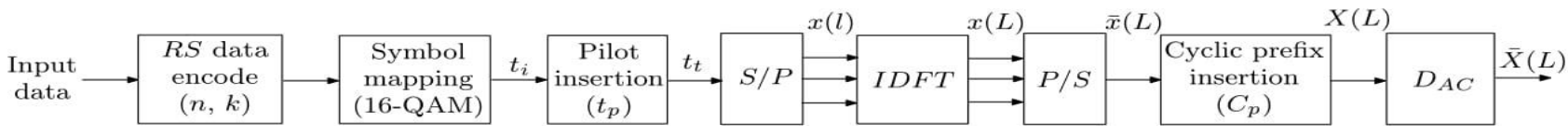

Figure 3: OFDM transmitter system model on time-varying frequency-selective Rayleigh fading channels.

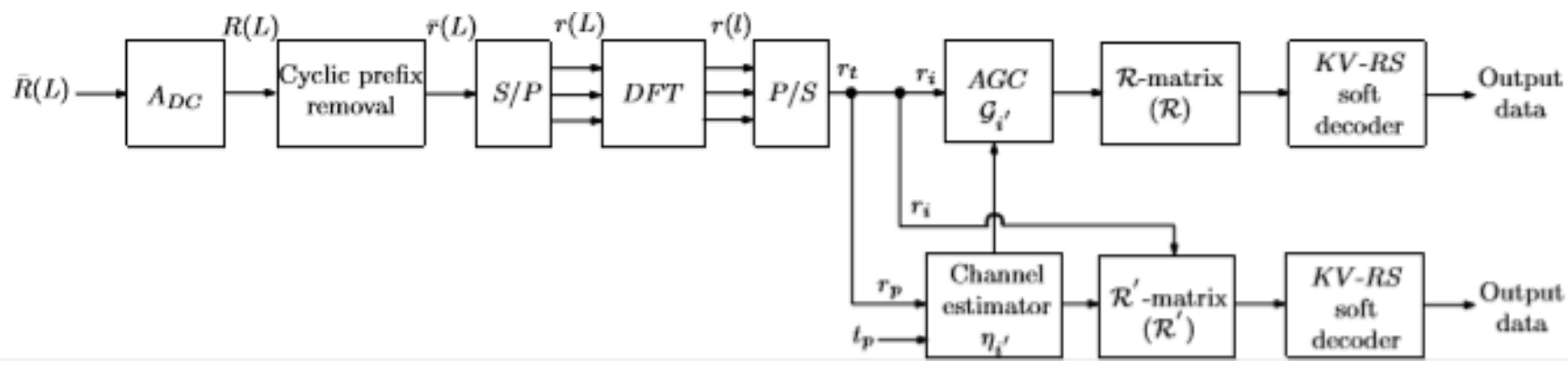

Figure 4: OFDM receiver system model on time-varying frequency-selective Rayleigh fading channels. 


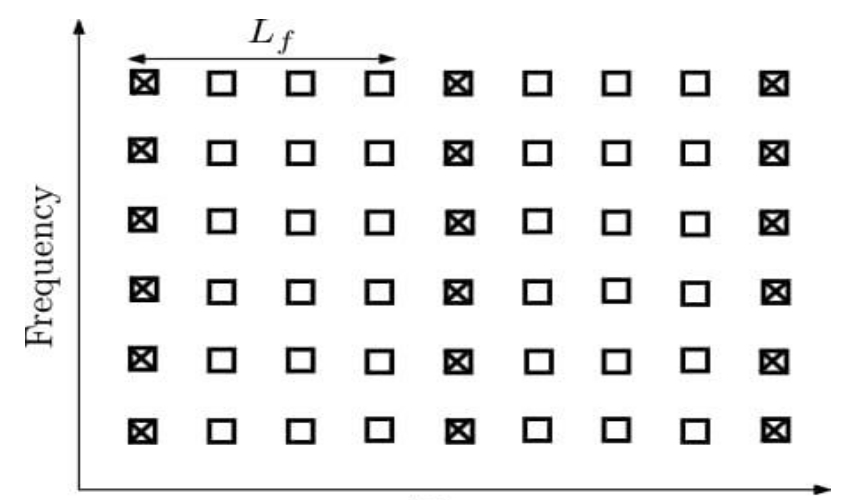

\section{Time \\ 口 Data symbol $\quad$ Pilot symbol \\ Figure 5: Block type OFDM pilot arrangement.}

The channel impulse response is defined with respect to the normalized Doppler frequency $f_{d} T$ as [10]:

$$
\bar{H}(L)=\sum_{\eta=0}^{\gamma-1} \bar{H}_{\eta} e^{j\left(2 \pi f_{d} T L / \lambda\right)} \delta\left(\varnothing-\psi_{\eta}\right),
$$

where $\bar{H}_{\eta}$ is the complex impulse response of the $\eta t h$ propagation path, $\psi_{\eta}$ is the $\eta t h$ path delay, $\emptyset$ is the average path gain, $\gamma$ is the number of propagation path. After the Analogue-to-Digital Conversion( $A_{D C}$ ) block, the cyclic prefix is removed. Therefore, the resulting time domain OFDM signal is defined as [10]:

$$
\bar{r}(L)=\left\{\begin{array}{lc}
\bar{r}(L), & -C_{p} \leq L \leq C_{p}-1, \\
\bar{r}\left(L+C_{p}\right) & L=0,1, \cdots, \lambda-1
\end{array}\right.
$$

The $\bar{r}(L)$ passes through the $S / P$ block followed by the DFT block. The DFT block converts the time domain OFDM signal $r(L)$ to the frequency domain as expressed in Eqn. (7) [10]:

$$
r(l)=\frac{1}{\sqrt{\lambda}} \sum_{L=0}^{\lambda-1} r(L) e^{-j(2 \pi l L / \lambda)} .
$$

Assuming the cyclic prefix eliminates the ISI, the received OFDM signal after the $P / S$ block is defined as:

$$
r_{t}=\kappa t_{t} \eta_{\mathrm{t}}+\mathrm{z}_{t}
$$

where the notation $\kappa$ is the matrix of the DFT defined as [10]:

$$
\kappa=\left[\begin{array}{ccc}
z_{\lambda}^{00} & \cdots & z_{\lambda}^{0(\lambda-1)} \\
\vdots & \ddots & \vdots \\
z_{\lambda}^{(\lambda-1) 0} & \cdots & z_{\lambda}^{(\lambda-1)(\lambda-1)}
\end{array}\right]
$$

with corresponding weight defined as [10]:

$$
z_{\lambda}^{l L}=\frac{1}{\sqrt{\lambda}} e^{-j(2 \pi l L / \lambda)} .
$$

$\eta_{\mathrm{t}}$ is the frequency domain CSI at the $t_{\mathrm{t}}$ symbol positions. Likewise, the CSI is known only at the pilot symbol positions. Thus, the CSI at the data symbol positions are derived from the properties of the known pilot symbols.

\section{CSI DERIVATION}

In this Section, we briefly derive the CSI over flat Rayleigh fading channels, and on time-varying frequency-selective Rayleigh fading channels. The channel estimation techniques are based on the LMMSE criterion and the cubic interpolation channel estimation method.

\subsection{LMMSE Estimator}

The observation at the pilot symbol position is defined as:

$$
r_{p}=t_{p} \eta_{p}+Z_{p}
$$

where $r_{p}, t_{p}, \eta_{p}$, and $Z_{p}$ are defined as above in terms of the pilot symbols. Thus, the CSI estimate at the data symbol positions using the LMMSE estimator is defined as [11]:

$$
\eta_{i}=H_{i}^{\dagger} r_{p}
$$

where the $\dagger$ sign denotes the conjugate transpose. The coefficient $H_{i}^{\dagger}$ is found by solving the Wiener-Hopf equation defined as [11]:

$$
C_{i}=\mathcal{A} H_{i} .
$$

The autocorrelation matrix $\mathcal{A}$, and the covariance vectors $C_{i}$ are defined by Eqn. (14) [11]:

$$
\mathcal{A}=E\left[r_{p} r_{p}^{\dagger}\right] \quad \text { and } \quad \mathrm{C}_{\mathrm{i}}=\mathrm{E}\left[\mathrm{H}_{\mathrm{p}}^{*} \mathrm{r}_{\mathrm{p}}\right]
$$

The $*$ sign denotes the complex conjugate. The coefficient $H_{i}$ is interpolated to obtain the CSI $\eta_{i}$ at each data symbol position. Reference [11] gives straightforward derivations of the LMMSE channel estimator. In a similar way, provided the channel vector $H_{p}$ is Gaussian and uncorrected with the channel noise $Z_{p}$, the frequency domain CSI estimate $H_{i}$ at the data symbol positions using the LMMSE estimator can be derived as $[10,11]$ :

$$
H_{i^{\prime}}=\kappa H_{i}^{\dagger} r_{p}
$$

All parameters are defined as in above but in the frequency domain.

\subsection{Cubic Interpolation Channel Estimator}

Consider the block type pilot arrangement shown in Fig.2. Let the four nearest pilot symbols CSI to the $\tau t h$ data symbol position be defined as $\left(H_{p}, \ldots, H_{p+3}\right)$, and the known positions of these four nearest pilot symbols be $\left(\delta_{p}, \delta_{p+1}, \delta_{p+2}, \delta_{p+3}\right)$ respectively. The CSI estimate using the cubic interpolation channel estimation technique is defined as [12]:

$$
\begin{array}{r}
H_{i}=\sum_{\tau=1}^{L_{f}-1} v_{1}+v_{2}(\tau)+v_{3}(\tau)^{2}+v_{4}(\tau)^{3} \\
\tau=1, \cdots, L_{f}-1
\end{array}
$$


where $\left(v_{1}, \ldots, v_{4}\right)$ are the cubic filter coefficients which are defined as [12]:

$$
\left[\begin{array}{l}
v_{1} \\
v_{2} \\
v_{3} \\
v_{4}
\end{array}\right]=\left[\begin{array}{cccr}
1 & \delta_{p} & \delta_{p}^{2} & \delta_{p}^{3} \\
1 & \delta_{p+1} & \delta_{p+1}^{2} & \delta_{p+1}^{3} \\
1 & \delta_{p+2} & \delta_{p+2}^{2} & \delta_{p+2}^{3} \\
1 & \delta_{p+3} & \delta_{p+3}^{2} & \delta_{p+3}^{3}
\end{array}\right]\left[\begin{array}{c}
H_{p} \\
H_{p+1} \\
H_{p+2} \\
H_{p+3}
\end{array}\right]
$$

Refer to reference [12] for straightforward derivations of the cubic channel estimator. Similarly, the frequency domain CSI is derive using the cubic estimator as:

$$
\begin{array}{r}
H_{i^{\prime}}=\kappa\left(\sum_{\tau=1}^{L_{f}-1} v_{1}+v_{2}(\tau)+v_{3}(\tau)^{2}+v_{4}(\tau)^{3}\right), \quad \text { (18) } \\
\tau=1, \cdots, L_{f}-1,
\end{array}
$$

where all parameters are also defined as in above but in the frequency domain.

\section{RELIABILITY MATRIX}

Conventionally, the estimated CSI is used to scale the received signal in a process called Automatic Gain Control (AGC) in order to compensate for the fading distortion which results due to the varying properties of the channel. Therefore, the soft reliability information ( $R$-matrix) is derived in the form of a DM from the scaled received signal and the symbols in the output signal constellation [6-8]. On the other hand, the soft reliability information $\left(R^{\prime}\right.$-matrix) can be derived from the estimated CSI and the derived scaled CSI as we propose in this work.

\subsection{Conventional Method}

Let the noisy received signal $r_{i}$ and the estimated CSI be defined by Eqn. (19) and Eqn. (20) respectively.

$$
\begin{aligned}
r_{i} & =\left[r_{0}, r_{1}, \ldots, r_{n-1}\right], \\
H_{i} & =\left[H_{0}, H_{1}, \ldots, H_{n-1}\right] .
\end{aligned}
$$

The estimated CSI $H_{i}$ is firstly used to scale the noisy received signal $r_{i}$ to derive the scaled received signal defined as:

$$
\mathcal{G}_{i}=\frac{r_{i}}{H_{i}} .
$$

The reliability matrix ( $R$-matrix) is therefore derived in the form of a DM from the scaled received signal $\mathcal{G}_{i}$, and the symbols in the output signal constellation as [7]:

$$
\mathcal{D}=\sqrt{\left(\mathcal{G}_{i_{x}}-\mathcal{Q}_{x}\right)^{2}+\left(\mathcal{G}_{i_{y}}-\mathcal{Q}_{y}\right)^{2}},
$$

where $Q_{x y}=\left(Q_{x}, Q_{y}\right)$ are the symbols in the output signal constellation. The in-phase and quadrature parts of the scaled received signal $\mathcal{G}_{i}$ are defined as $\mathcal{G}_{i_{x}}$ and $\mathcal{G}_{i y}$ respectively.

In Eqn. (22), the distance from the scaled received signal $\mathcal{G}_{i}$ to all the output signal constellation points $Q_{x y}$ are measured to form an $M \times n R$-matrix $(R)$ defined as [6-8]:

$$
\left[\begin{array}{cccc}
\mu_{0,0} & \mu_{0,1} & \cdots & \mu_{0, n-1} \\
\mu_{1,0} & \mu_{0,2} & \cdots & \mu_{1, n-1} \\
\vdots & \vdots & \ddots & \vdots \\
\mu_{M-1,0} & \mu_{M-1,1} & \cdots & \mu_{M-1, n-1}
\end{array}\right]
$$

where $\mu_{M-1, n-1}$ is the reliability value representing the likelihood of receiving a symbol before soft decoding. The entries of $R$ in Eqn. (23) are normalized and quantized to $\log _{2}(M)$ bits of precision.

In a similar approach, the estimated frequency domain CSI $H_{i^{\prime}}$ is used to scale the received noisy received signal $r_{i}$ to obtain the scaled received frequency domain signal defined as:

$$
\mathcal{G}_{i^{\prime}}=\frac{r_{i}}{H_{i^{\prime}}}
$$

Thus, the reliability matrix ( $R$-matrix) is also derived in the form of a DM from the scaled received frequency domain signal $\mathcal{G}_{i^{\prime}}$, and the symbols in the output signal constellation as [7]:

$$
\mathcal{D}=\sqrt{\left(\mathcal{G}_{i_{x}^{\prime}}-\mathcal{Q}_{x}\right)^{2}+\left(\mathcal{G}_{i_{y}^{\prime}}-\mathcal{Q}_{y}\right)^{2}},
$$

where the in-phase and quadrature parts of the scaled received frequency domain signal $\mathcal{G}_{i^{\prime}}$ are defined as $\mathcal{G}_{i_{x}^{\prime}}$ and $\mathcal{G}_{i_{y}^{\prime}}$ respectively. Hence, Eqn. (25) will produce a similar $M \times n R$-matrix as Eqn. (23).

\subsection{Proposed Method}

We propose an improved method of extracting reliability information over fading channels in the form of a distance metric. This approach computes the soft reliability information directly from the estimated CSI $\square_{i}$ and the derived scaled CSI. The scaled CSI is first derived in a matrix form as:

$$
\beta_{i}=\left\{\beta_{u, w}\right\} \text {. }
$$

The notation $\beta_{u, w}$ is defined in terms of the noisy received signal $r_{i}$ and the output signal constellation points $Q_{x y}$ as:

$$
\begin{gathered}
\beta_{u, w}=\frac{r_{i_{w}}}{Q_{x y_{u}}}, \quad \begin{array}{l}
u=0, \cdots, M-1 \quad \text { and } w \\
=
\end{array}, \cdots, n-1 .
\end{gathered}
$$

The reliability information matrix $\left(R^{\prime}\right.$-matrix) is therefore derived from the scaled CSI $\beta_{i}$, and the estimated CSI $\eta_{i}$ in the form of a DM as:

$$
\mathcal{D}^{\prime}=\sqrt{\left(\beta_{i_{x}}-\eta_{i_{x}}\right)^{2}+\left(\beta_{i_{y}}-\eta_{i_{y}}\right)^{2}},
$$

where the in-phase and quadrature parts of the estimated CSI $\eta_{i}$ are denoted as $\eta_{i_{x}}$ and $\eta_{i_{y}}$ respectively. Likewise, $\beta_{i_{x}}$ and $\beta_{i_{y}}$ are the in-phase and quadrature parts of the scaled CSI $\beta_{i}$ respectively. In Eqn. (28), the distance from the estimated CSI $\eta_{i}$ to all the entries in the scaled CSI $\beta_{i}$ are measured to form an $M \times n R^{\prime}$ matrix $\left(R^{\prime}\right)$ defined as: 


$$
\left[\begin{array}{cccc}
\mu_{0,0}^{\prime} & \mu_{0,1}^{\prime} & \cdots & \mu_{0, n-1}^{\prime} \\
\mu_{0,1}^{\prime} & \mu_{0,2}^{\prime} & \cdots & \mu_{1, n-1}^{\prime} \\
\vdots & \vdots & \ddots & \vdots \\
\mu_{M-1,0}^{\prime} & \mu_{M-1,1}^{\prime} & \cdots & \mu_{M-1, n-1}^{\prime}
\end{array}\right]
$$

where $\mu_{M-1, n-1}^{\prime}$ is the reliability value representing the likelihood of receiving a symbol before soft decoding. Also, the entries of $R^{\prime}$ - in Eqn. (29) are normalized and quantized to $\log _{2}(M)$ bits of precision. In the same way, we derive the scaled frequency domain CSI as:

$$
\beta_{i^{\prime}}=\left\{\beta_{(u, w)^{\prime}}\right\} \text {. }
$$

where $\beta_{(u, w)^{\prime}}$ is also defined in terms of the noisy received signal $r_{i}$ and the output $M$-QAM constellation points $\mathcal{Q}_{x y}$ as:

$$
\begin{gathered}
\beta_{(u, w)^{\prime}}=\frac{r_{i_{w}}}{Q_{x y_{u}}}, \quad u=0, \cdots, M-1 \text { and } w \\
=0, \cdots, n-1 .
\end{gathered}
$$

The reliability matrix ( $R^{\prime}$-matrix) is thus derived in the form of a DM from the scaled frequency domain $\operatorname{CSI} \beta_{i^{\prime}}$, and the estimated frequency domain CSI ${ }_{i}{ }_{i}$ as:

$$
\mathcal{D}^{\prime}=\sqrt{\left(\beta_{i_{x}^{\prime}}-\eta_{i_{x}^{\prime}}\right)^{2}+\left(\beta_{i_{y}^{\prime}}-\eta_{i_{y}^{\prime}}\right)^{2}},
$$

where the in-phase and quadrature parts of the estimated frequency domain CSI $\eta_{i^{\prime}}$ are denoted as $\eta_{i_{x}^{\prime}}$ and $\eta_{i y}^{\prime}$ respectively. The in-phase and quadrature parts of the scaled frequency domain CSI $\beta_{i^{\prime}}$ are represented by $\beta_{i_{x}^{\prime}}$ and $\beta_{i_{y}^{\prime}}$ respectively. As such, Eqn. (32) will produce a similar $M \times n R$-matrix as Eqn. (29).

\section{PERFORMANCE ANALYSIS}

\subsection{Flat Rayleigh Fading Channels}

A computer simulation study was carried out to investigate the Codeword Error Rate (CER) performance of the conventional and proposed DM methods on flat Rayleigh fading channels using $M$-QAM. For the same input data, the reliability information derived in Eqn. (23) and Eqn. (29) are fed into the KV$R S$ soft decision decoder. The KV- $R S$ algorithm converts this reliability information into a choice set of interpolation points along with their multiplicities. The KV-RS decoding algorithm builds upon the interpolation and factorization steps of the Guruswami and Sudan algorithm [15]. An $R S$ codeword length $\mathrm{n}=15$, and information symbol length $k=7$ are assumed for the $M$-QAM transmission design of Fig. 1. A list-size $L_{s}=4$ is used for the KV-RS soft decoder in order to reduce the simulation delay and time complexity. The list size $L_{s}\left(L_{s} \geq 1\right)$ determines the decoding performance of the KV-RS soft decision decoder. The higher the value $L_{S}$ the better the decoding performance of the KV-RS soft decoding algorithm; however, it comes with a price of high decoding time complexity. Refer to $[6,15,16]$ for more details on the KV- $R S$ algorithm.

Frame length sizes $L_{f}=4$ and $L_{f}=6$ are assumed for PSAM LMMSE and cubic estimators. Two normalized Doppler frequencies $\left(f_{d} T\right)$ are considered in this work: $f_{d} T=0.010$ and $f_{d} T=0.015$. Fig. 6 and Fig. 7 show the CER performance of the proposed DM method in comparison to the conventional DM method analysed in [6-8] for these normalized Doppler frequencies. From the figures, the proposed DM method of extracting soft reliability information exhibits superior CER performance in comparison to the conventional DM method. As also shown in the figures, the CER performance of both methods of extracting reliability information over fading channels drop with increase in the normalized Doppler $f_{d} T$; nevertheless, the proposed DM method displays superior CER performance to the conventional DM method. The CSI is difficult to obtain as the fading rate $\left(f_{d} T\right)$ of the channel increases; therefore, the reliability information metric derived in Eqn. (23) and Eqn. (29) using the conventional and proposed DM methods respectively are not accurate. This resulted in the noticeable reduction in the CER performance as the fading rate increases from $f_{d} T=0.010$ to $f_{d} T=0.015$. Furthermore, as the frame length size $L_{f}$ increases from $L_{f}=4$ to $L_{f}=6$, the CER performance of both DM methods degrade but the proposed DM method displays significant improvement in CER performance compared to the conventional DM method. More so, as shown in Fig. 6 and Fig. 7, irrespective of the channel estimation technique used, the proposed DM method offers significant improvement in CER performance $(+0.5 \mathrm{~dB})$ in comparison with the conventional DM method. As a note, the performance of the PSAM LMMSE and cubic channel estimators depend on the frame length $L_{f}$. The smaller the frame length size, the better the performance of the estimators.

\subsection{Time-Varying Frequency-selective Rayleigh Fading Channels}

We also investigate the CER performance of the proposed and conventional DM methods on timevarying frequency-selective Rayleigh fading channels employing OFDM systems. The OFDM system parameters used in the computer simulation is as shown in Table 1. Perfect synchronization is assumed in the simulation design in order to prevent ISI and Inter Carrier Interference (ICI). We select the cyclic prefix to be $10 \%$ of the transmitted OFDM symbol duration such that the maximum average path gain is less than this chosen cyclic prefix. 


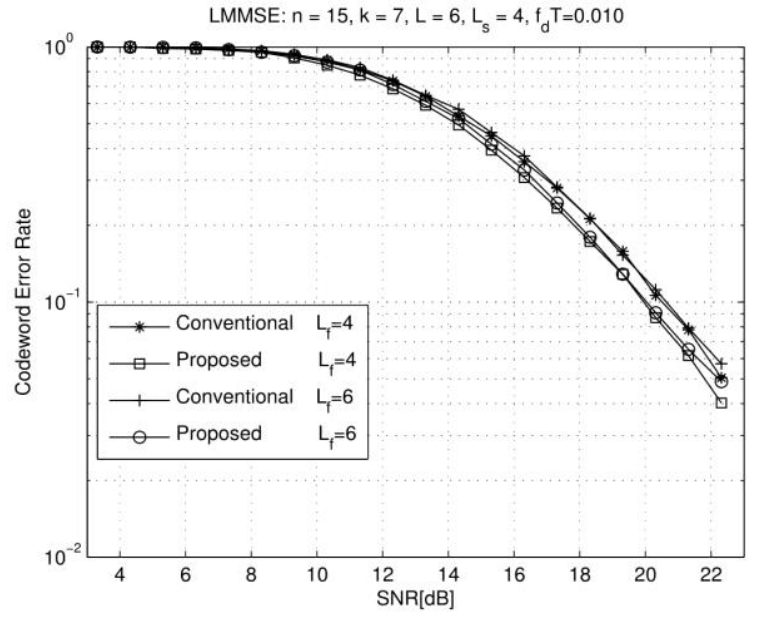

(a) $f_{d} T=0.010$

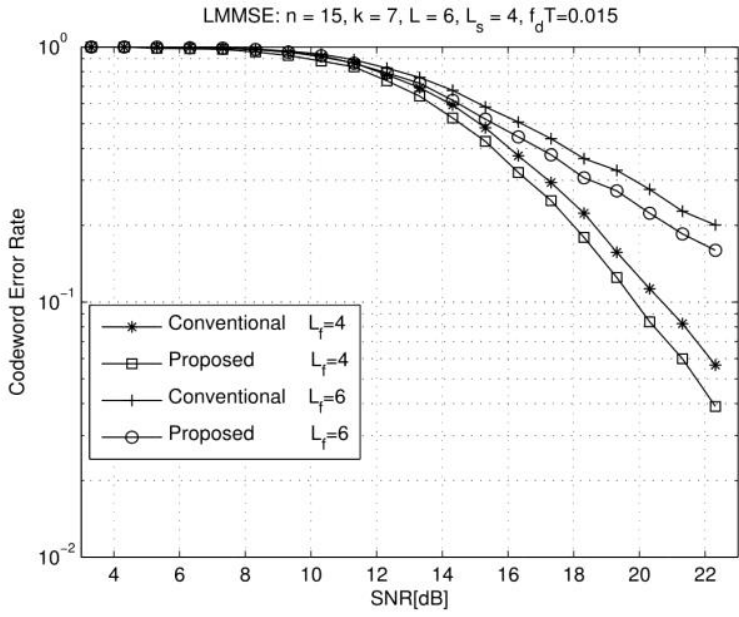

(b) $f_{d} T=0.015$

Figure 6: M-QAM system performance in terms of CER versus SNR for LMMSE estimator.

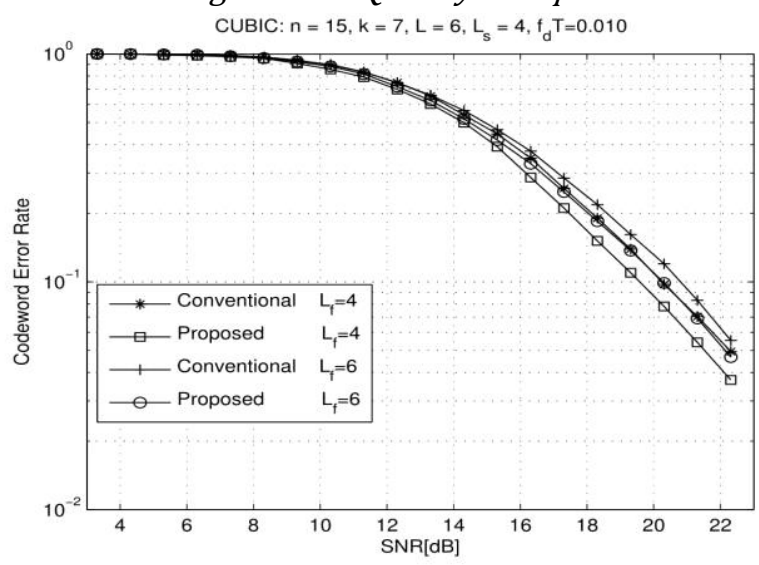

(a) $f_{d} T=0.010$

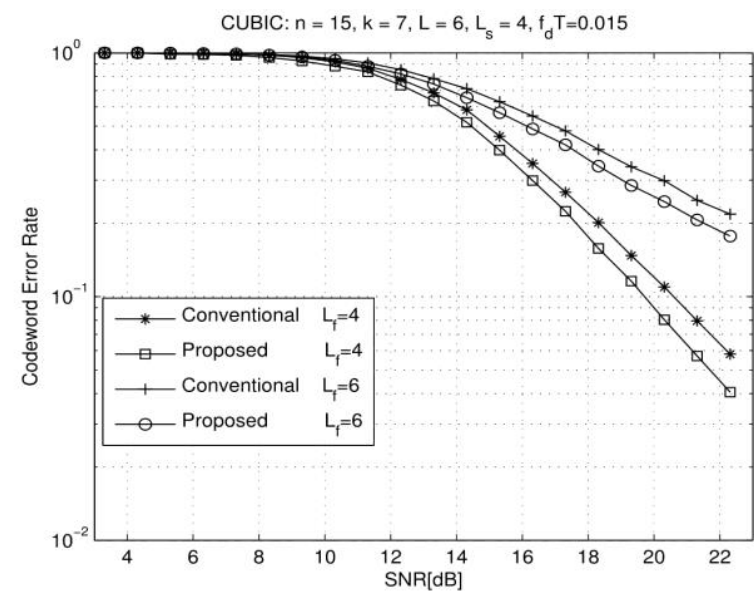

(b) $f_{d} T=0.015$

Figure 7: M-QAM system performance in terms of CER versus SNR for cubic estimator.

Table 1: OFDM simulation parameters

\begin{tabular}{lc}
\hline Parameters & Specifications \\
\hline FFT size & 64 \\
Coding rate, $k / n$ & $7 / 15$ \\
List size of KV-RS & 4 \\
algorithm, $L_{s}$ & $4 / 15,6 / 15$ \\
Pilot to data symbol ratio & $10 \mathrm{kHz}$ \\
Sampling rate & $16-\mathrm{QAM}$ \\
Signal constellation & $0.010,0.015$ \\
Normalized Doppler $f_{d} T$ & Rayleigh Fading, \\
Channel model & Multipath \\
\hline
\end{tabular}

Four taps or multipath are used in the simulation set up, such that the first tap always have a zero path delay which closely matches a perfect synchronization.

For the same input data, the derived reliability information matrices are also fed into the KV-RS soft decision decoder to verify their performance on timevarying frequency-selective Rayleigh fading channels. Similarly, frame length sizes $L_{f}=4$ and $L_{f}=6$ are defined for the PSAM LMMSE and cubic channel estimator assuming a normalized Doppler of $f_{d} T=0.010$ and $f_{d} T=0.015$. Fig. 8 and Fig. 9 show the CER performance of the proposed DM method compared to the conventional DM method. From the figures, the proposed DM method of extracting soft reliability information on time-varying frequencyselective Rayleigh fading channels display superior CER performance in comparison to the conventional DM method even at moderately low Signal-to-Noise Ratios (SNR). Furthermore, the proposed DM method exhibits superior CER performance irrespective of the frame length size and channel estimation technique employed as shown in Fig. 8 and Fig. 9.

In general, the proposed DM method of extracting reliability information over fading channels introduces little or no bias in deriving the reliability values for each output symbol. It allocates the closest symbols to the received constellation point with almost the same reliability value. Therefore, it gives the FEC soft 
decoder enough freedom to prioritize more symbols with good reliability values. The conventional DM method on the other hand assigns a high reliability value to the potential symbol compared to other closeby symbols on the output constellation. This may result in a decoding failure if the potential symbol with high reliability value is not the transmitted symbol. The FEC soft decoder in this case has less symbols to prioritize; thus, this degrades the decoding performance of the soft FEC decoder.

\subsection{Comparative Computational Complexity Analysis of the Conventional and Proposed DM Methods}

In terms of computational time complexity comparison, the conventional DM method will require $3 M n$ additional/subtraction operations, $n$ multiplication/division operations, $2 M n$ square operations, and $M n$ square root operations. The proposed DM method on the other hand will require $3 M n$ additional/ subtraction operations, $M n$ multiplication/division operations, $2 M n$ square

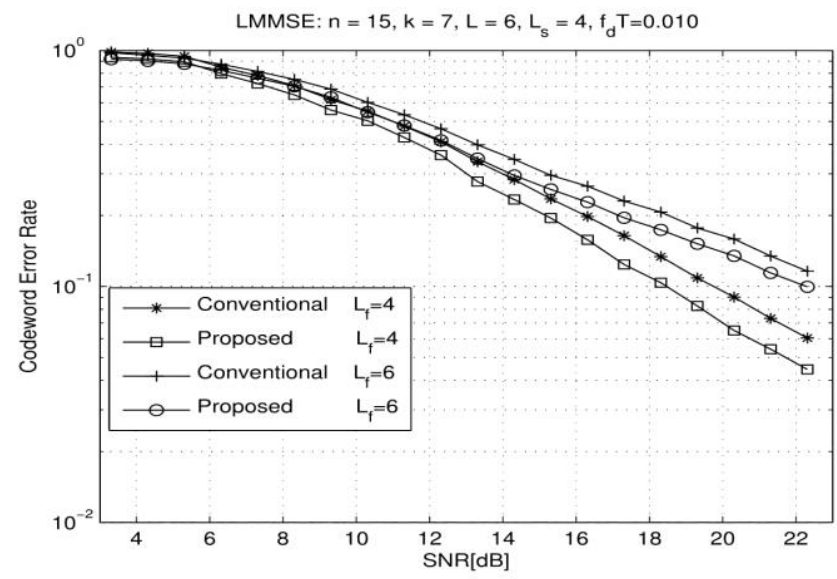

(a) $f_{d} T=0.010$

Figure 8: OFDM system performance in terms of CER versus SNR for LMMSE estimator.

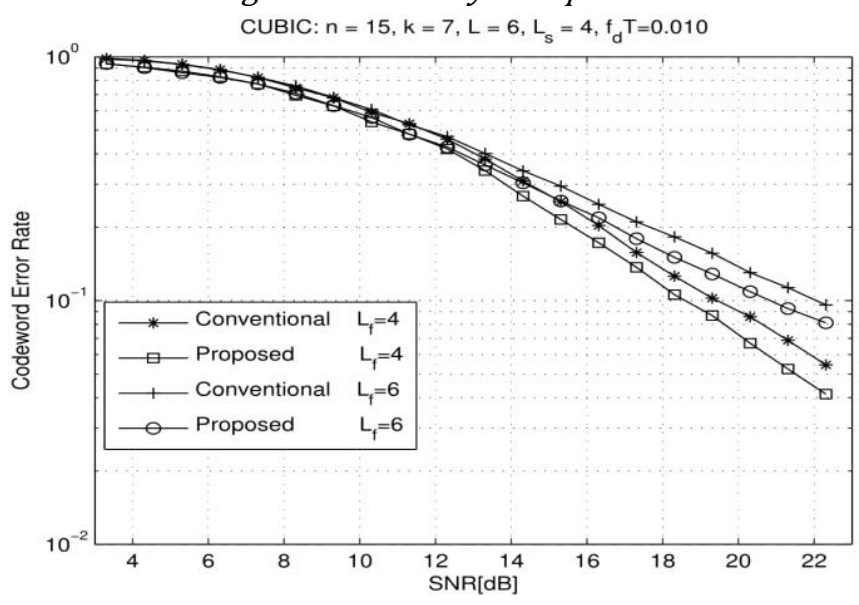

(a) $f_{d} T=0.010$ operations, and $M n$ square root operations as shown in Table 2.

From Table 2, given $M=16$, and $n=15$, only the total number of multiplication/division operations required using the conventional DM method to produce Eqn. (23) is higher in comparison to the multiplication/division operations required using the proposed DM method to produce Eqn. (29). From these analysis, it can be concluded that the proposed DM method displays higher computational time complexity in comparison with the conventional DM method; however, both DM methods can be classified under the $(O(M n))$ time complexity order. Thus the proposed DM method can be used on real time modulation systems . Also, considering the CER performance improvement displayed by this proposed DM method as shown in Fig. 6 to Fig. 9, we can conclude that this proposed method proffers a balance in the computational delay and time complexity, and the modulation system's gain performance.

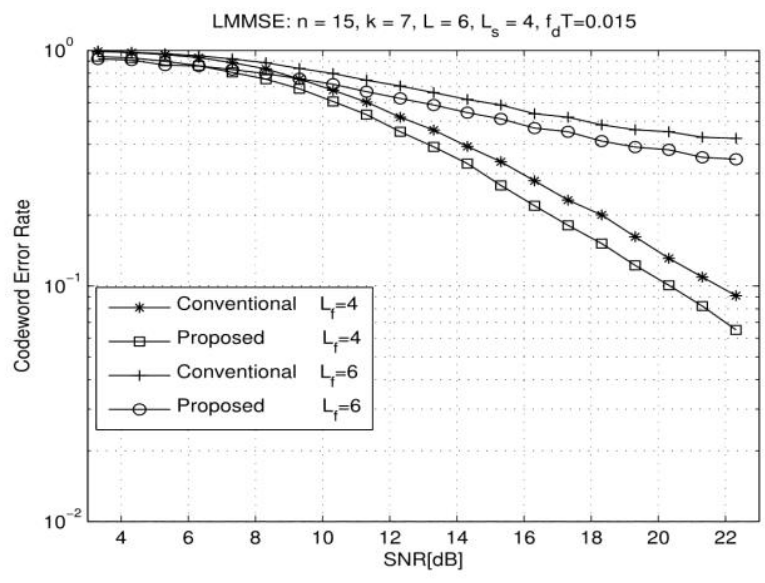

(b) $f_{d} T=0.015$

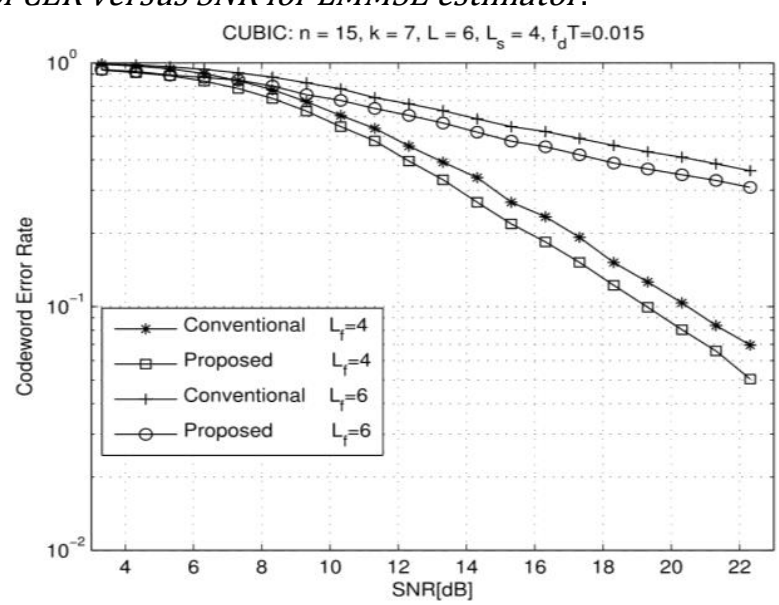

(b) $f_{d} T=0.015$

Figure 9: OFDM system performance in terms of CER versus SNR for cubic estimator.

Table 2: Computational complexity per iteration 


\begin{tabular}{c|c|c|c|c}
\hline \multirow{2}{*}{ Operations } & \multicolumn{2}{|c|}{ Conventional } & \multicolumn{2}{c}{ Proposed } \\
\cline { 2 - 5 }$+/-$ & $3 M n$ & $M=16, n=15$ & $3 M n$ & $M=16, n=15$ \\
$\times / \div$ & $n$ & 720 & $M n$ & 240 \\
\{\}$^{2}$ & $2 M n$ & 480 & $2 M n$ & 480 \\
$\sqrt{ }$ & $M n$ & 240 & $M n$ & 240 \\
\hline
\end{tabular}

\section{CONCLUSION}

This paper has studied an improved Distance Metric (DM) method of deriving soft reliability information over Rayleigh fading channels. The reliability information is computed from the estimated CSI and the scaled CSI. We presented the new metric CER performance and a fair comparison with the conventionally used DM method. Also, a simple comparative computational time complexities between the conventional and the proposed DM method is presented in the paper. Firstly, the proposed DM method displayed significant CER performance in comparison to the conventional DM method even at low SNR. Fig. 6 to Fig. 9 confirms our analysis for both flat Rayleigh fading channels and time-varying frequency-selecting Rayleigh fading channels. Secondly, the proposed DM method slightly increase the computational delay and time complexity of the overall system design in comparison to the conventional DM method as shown in Table 2. However, considering the significant improvement in CER performance offered by the proposed DM method, this method offers suitable trade-off between the computational delay and time complexity, and the gain performance of the systems. Lastly, the proposed DM method performance do not depend on the channel estimation technique used in computing the CSI. Computer simulation results presented in Fig. 6 to Fig. 9 assert our analysis for PSAM LMMSE and cubic estimators.

\section{ACKNOWLEGEMENT}

The authors acknowledge the contributions of Dr D.J.J. Versfeld (Department of Electrical and Electronic Engineering, Stellenbosch University, South Africa) towards this research work.

\section{REFERENCES}

[1] O. Oyerinde and S. Mneney, "Iterative receiver with soft-input-based-channel estimation for orthogonal frequency division multiplexinginterleave division multiple access systems," Communications, IET, vol. 8 , no. 14 , pp. $2445-$ 2457, Sept 2014.
[2] Y. Jiang, A. Ashikhmin, and N. Sharma, "LDPC Codes for Flat Rayleigh Fading Channels with Channel Side Information," Communications, IEEE Transactions on, vol. 56, no. 8, pp. 1207-1213, August 2008.

[3] K. Fu and A. Anastasopoulos, "Analysis and design of LDPC codes for time-selective complex-fading channels," IEEE Transactions on Wireless Communications, , vol. 4, no. 3, pp. 1175-1185, May 2005.

[4] D. Feng Yuan and X.-F. Song, "Turbo code performance over Rayleigh fading channel using QPSK modulation," in TENCON '02. Proceedings, 2002 IEEE Region 10 Conference on Computers, Communications, Control and Power Engineering, vol. 2, Oct, pp. 1056-1059 vol.2, 2002.

[5] G. Van Meerbergen, M. Moonen, and H. De Man, "CTH03-3: Reed-Solomon Codes Implementing a Coded OFDM Scheme for Rayleigh Fading Channels," in Global Telecommunications Conference, 2006. GLOBECOM '06. IEEE, Nov 2006, pp. 1-6.

[6] R. Koetter and A. Vardy, "Algebraic soft-decision decoding of Reed-Solomon codes," Information Theory, IEEE Transactions on, vol. 49, no. 11, pp. 2809-2825, Nov 2003.

[7] W. Phoel, J. Pursley, M. Pursley, and J. Skinner, "Frequency-hop spread spectrum with quadrature amplitude modulation and errorcontrol coding," in Military Communications Conference, 2004. MILCOM 2004. 2004 IEEE, vol. 2, Oct, pp. 913-919 Vol. 2, 2004.

[8] T. K. Moon, Error Correction Coding: Mathematical Methods and Algorithms. John Wiley \& Sons, 2005.

[9] S. Allpress, C. Luschi, and S. Felix, "Exact and approximated expressions of the log-likelihood ratio for 16-QAM signals," in Signals, Systems and Computers, 2004. Conference Record of the Thirty-Eighth Asilomar Conference on, vol. 1, Nov, pp. 794-798 Vol.1. 2004.

[10] S. Coleri, M. Ergen, A. Puri, and A. Bahai, "Channel estimation techniques based on pilot arrangement in OFDM systems," IEEE Transactions on 
Broadcasting, , vol. 48, no. 3, pp. 223-229, Sep 2002.

[11] J. Cavers, "An analysis of pilot symbol assisted modulation for Rayleigh fading channels [mobile radio]," IEEE Transactions on Vehicular Technology, vol. 40, no. 4, pp. 686-693, Nov 1991.

[12] L. Erup, F. M. Gardner, and R. Harris, "Interpolation in digital modems. ii. implementation and performance," IEEE Transactions on Communications, vol. 41, no. 6, pp. 998-1008, Jun 1993.

[13] M. K. Simon and J. Smith, "Carrier Synchronization and Detection of QASK Signal Sets," EEE
Transactions on Communications, I, vol. 22, no. 2, pp. 98-106, Feb 1974.

[14] W. C. Jakes, Mobile microwave communication. New York: Wiley, 1974.

[15] V. Guruswami and M. Sudan, "Improved decoding of Reed-Solomon and algebraic-geometry codes,", IEEE Transactions on Information Theory, vol. 45, no. 6, pp. 1757-1767, Sep 1999.

[16] L. Chen and R. Carrasco, "Soft decoding of algebraic-geometric codes using Koetter-Vardy algorithm," Electronics Letters, vol. 45, no. 25, pp. 1334-1336, December 2009. 